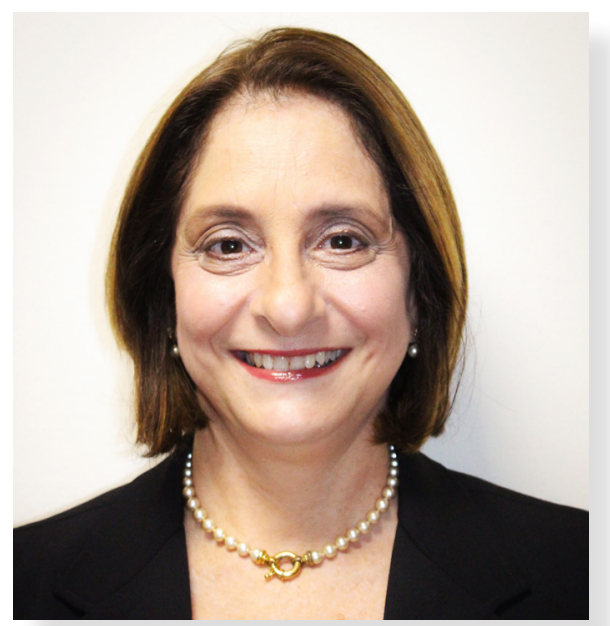

Jeanette Chaljub Hasbún*

\section{El b-learning y la clase invertida para el desarrollo del aprendizaje activo, la autogestión y el pensamiento crítico en el ámbito universitario}

\section{B-learning and flipped classroom as tools for the development of active learning, self-management and critical thinking in higher education}

Recibido: 19-03-17 Aprobado: 17-04-17

\title{
Resumen
}

Esta investigación está enfocada en el método de Clase Invertida o Flipped Classroom (FC), la cual combina los aprendizajes individuales con las construcciones colectivas de conocimientos. El objetivo principal de este estudio es desarrollar la autogestión y el pensamiento crítico a través de la clase invertida y trabajo colaborativo en estudiantes de la Carrera de Educación. Resalta la importancia de hacer una transferencia metodológica del modelo centrado en el profesor a aquel centrado en los aprendizajes. Contempla una metodología bimodal, en la que se diseña una situación de enseñanza para que los alumnos puedan ir aprendiendo conceptos y estrategias nuevas antes de la clase, por medio de vídeos y lecturas de documentos (habilidades cognitivas inferiores). Luego, en presencia del docente, se desarrollan ejercicios y actividades para poner en práctica habilidades cognitivas superiores de forma colaborativa y con una atención más cercana del profesor. Este estudio presenta una investigación de corte transversal, con un alcance interpretativo. Entre los hallazgos obtenidos se encuentran la valoración positiva de la clase invertida y la potenciación del desarrollo de los aprendizajes activos, tanto de forma individual como colectiva.

\section{Abstract}

Flipped classroom (FC) is the main focus of this proposal. The individual knowledge is combined with group learning, and the main idea is to transfer teacher centered classes to student centered strategies. The main objective is to develop critical thinking and self-learning through flipped classroom and collaborative work among Education students. It also presents the concept of blended learning in which the apprentices study a topic, thru videos and documents (lower cognitive level), before the class begins. Once in the classroom, the teacher can develop learning exercises and activities to solving problems (higher cognitive level). He/she can also pay more attention to the students, both individually and in groups. This is a cross-sectional action-research. It has an interpretative scope that describes the student perception about Flipped Learning, and the way they believe it can help them to achieve their learning goals. The results show that the participant students value the flipped experience as positive, and they could build new knowledge as well as sharing with their classmates.

\section{Palabras clave}

clase invertida; trabajo colaborativo; aprendizaje combinado; recursos educativos abiertos

\section{Keywords}

flipped classroom; collaborative work; blended learning; open educational resources

\footnotetext{
*Jeanette M. Chaljub Hasbún: Doctora en Educación por la Universidad de Murcia, Master in Education, Major International Teaching por Framingham State College, Licenciada en Educación Básica por la Universidad de la Tercera Edad, Ingeniera Química por la Universidad Autónoma de Santo Domingo. Profesora a tiempo completo de la PUCMM. Para contactar a la autora: jeanettechaljub@pucmm.edu.do,jeannette.chaljub@gmail.com
} 


\section{Introducción}

A pesar del rápido y vertiginoso caudal de información que se genera segundo a segundo y de nuevas formas de pensar y modelos de actuar por todos los integrantes de la sociedad actual, las prácticas educativas siguen ancladas en métodos tradicionales y pasivos que, muchas veces, solo promueven repetición de conceptos, quedando en aprendizajes superficiales. Esto provoca el desinterés en los estudiantes, quienes manifiestan que las clases son "aburridas" y que no se sienten motivados, ya que el alumno generalmente es el receptor y el docente hace las veces del contenedor de todos los conocimientos.

Con el advenimiento de sociedades digitales y un mundo en constante cambio, donde prima la creatividad y la capacidad de enfrentar situaciones, en la mayoría de los casos no previsibles, es necesario que los espacios educativos modifiquen sus prácticas docentes, promoviendo la metodología activa donde el estudiante es el enfoque central de los procesos de enseñanza. Corroborando lo anterior, Maquilón, Mirete, García y Hernández (2013), afirman que:

Las tecnologías per sé no suponen un elemento innovador en la realidad de los estudiantes, pero sí pueden constituir una fuente de motivación siempre que se empleen desde una perspectiva y acción pedagógica que contribuya al desarrollo de procesos de enseñanza-aprendizaje efectivos y de calidad para el estudiante (p. 540).

Cabe destacar la necesidad de que los profesores, tanto a nivel pre-universitario como el universitario, busquen estrategias de enseñanza alternativas que promuevan el interés en los estudiantes, a través de la participación activa en la construcción de saberes. Para esto, el punto de inflexión ha de girar en torno al pensamiento crítico, la atención a la diversidad, la emoción, el aprendizaje autónomo, la investigación, la búsqueda de consenso con los pares, confrontación de ideas, etc., a través de metodologías innovadoras y novedosas que generan entusiasmo y un ambiente cómodo en la clase.

Cambiar la metodología, de una centrada en el docente, a una centrada en los aprendizajes, puede promover la motivación y la autogestión, haciendo que los estudiantes se comprometan con su propio proceso y logren la construcción individual y colectiva de los conocimientos. El carácter convencional que, hasta hace poco, se ha venido presentando en las aulas universitarias, "ha brindado escasas oportunidades para el aprendizaje activo en el aula, y eso a pesar de la evidencia empírica que permite concluir sobre la conveniencia de introducir oportunidades para aprender activamente en clase" (Prieto, 2006, p. 174). Un ejemplo de promoción de la participación activa se trata de la clase invertida o aprendizaje invertido, donde se cambia de un modelo tradicional, en el que las tareas se hacen en casa después de la explicación del profesor, a uno más interactivo y participativo, donde las tareas se realizan en clase, una vez que el estudiante haya estudiado de forma autónoma los conceptos básicos, con la guía y orientación del profesor. Esta estrategia tiene como característica principal el fomento del pensamiento crítico, además de la autogestión de los aprendizajes, potenciando la resolución de problemas, a través de la optimización del tiempo de aula (Domínguez, 2015).

De lo anterior se desprende que las estrategias utilizadas por el docente en un ambiente como el mencionado, han de ser lo suficientemente ricas en experiencias significativas por lo que demanda un cambio de paradigma hacia la consecución de nuevas estructuras de pensamiento. Esto, combinado con las tecnologías apropiadas, da lugar a lo que se conoce como aprendizaje mixto o blended learning.

\section{Objetivos}

\section{Objetivo general}

Desarrollar la autogestión de los aprendizajes y el pensamiento crítico a través de la clase invertida y trabajo colaborativo en estudiantes de la Carrera de Educación.

\section{Objetivos específicos}

- Relacionar el trabajo colaborativo con la clase invertida para potenciar la participación activa y la construcción colectiva de los conocimientos.

- Implementar nuevas herramientas y recursos telemáticos para la consecución de los aprendizajes.

- Evaluar cómo impacta este método en el desarrollo niveles de pensamiento superiores.

- Valorar la metodología y el grado de aceptación por parte de los estudiantes.

\section{Fundamentos teóricos}

Los estudiantes de hoy aprenden de manera distinta a como aprendimos hace unas décadas atrás. El impresionante caudal de información que surge día tras día, minuto a minuto, sugiere que busquemos alternativas didácticas que contribuyan a movilizar los aprendizajes activos. A través de la participación, la autorregulación de los aprendizajes, los estudiantes desarrollan 
destrezas que van desde la gestión de la información a la gestión de los conocimientos. Sobre esto, se destaca que el aprendizaje activo hace una transformación del conocimiento tácito y personal y se convierte en conocimiento explícito y compartido con otras personas (Gairín, 2007). De ahí, que las clases han de ser diseñadas regulando la sobrecarga de información para que se puedan construir conocimientos de forma colectiva.

Los aprendizajes intrapersonales, aquellos que son individuales, se complementan con los aportes de los compañeros para crear nuevos saberes, conectados con los saberes previos. Se aprende a través de la participación.

A pesar de que existe un espectro amplio de enfoques constructivas, este estudio se basa en el enfoque cognitivo, sustentado en la teoría piagetiana, en el que "el aprendizaje es, por tanto, un proceso interno que consiste en relacionar la nueva información con las representaciones preexistentes, lo que da lugar a la revisión, modificación, reorganización y diferenciación de esas representaciones" (Serrano \& Pons, 2011, pp. 5-7). Sin embargo, además de realizar un proceso interno, es importante la socialización de conocimientos e ideas sobre los temas a desarrollar, sobre todo, donde se evolucione de una clase centrada en la enseñanza a una centrada en los aprendizajes. Así lo corrobora McAlpine (2004), cuando propone que la incorporación de los alumnos en la actividad de clase, es el período clave para la calidad de los aprendizajes.

En el ámbito universitario, nuestros estudiantes necesitan desarrollar distintos tipos de conocimientos, destrezas y actitudes para poder insertarse a la sociedad actual. Del conjunto de competencias requeridas para profesionales del siglo XXI, planteadas por el Observatorio Navarro de Empleo en el 2012, podemos mencionar las siguientes habilidades, que están relacionadas de manera directa con la clase:

- Encontrar nuevas ideas y soluciones

- Utilizar herramientas informáticas

- Adquirir con rapidez nuevos conocimientos

- Coordinar actividades

- Predisposición para cuestionar ideas propias o ajenas

- De pensamiento analítico

- Hacer valer tu autoridad

- Movilizar las capacidades de otros

- Redactar informes o documentos

- Sintetizar y extraer conclusiones generales

Haciendo un recorrido por estas habilidades y perfiles cabe, entonces, resaltar la importancia que tiene la constante búsqueda de innovación pedagógica en la universidad. Es necesario que modifiquemos las estrategias de enseñanza y, con ellas, las actividades de evaluación.

En este mismo orden de ideas, este estudio combina la metodología colaborativa en la que subyace la estructura conversacional, asociada a una dinámica interactiva en el interior del grupo o interacción-social que, además, se ha de considerar compleja, tanto a nivel de Pregrado como en Postgrado (Maldondo, 2007; Pérez, Bustamante \& Maldonado, 2007), con dinámicas de aprendizaje activo para la construcción colectiva de los conocimientos.

El trabajo colaborativo propone una estrategia de enseñanza en la que todos los miembros de un equipo trabajan juntos para metas comunes, con responsabilidades compartidas e interdependencia positiva. Se aleja del típico modelo de trabajo cooperativo. Este último funciona en el sentido en que cada miembro es responsable de "una parte" del trabajo, que, luego, se pone en común. Desde la epistemología, el trabajo colaborativo se sustenta en el Constructivismo, ya que los miembros participan activamente en la construcción de saberes a través de la interacción con los demás (Constructivismo Social) y con su entorno. De allí que "el docente tiene una gran responsabilidad en alentar, promover y crear el espacio apropiado para la construcción del conocimiento" (Maldonado, 2007, p. 268). Se pasa de un aprendizaje pasivo a uno activo y autónomo.

En consonancia con las ideas planteadas, se hace imperativo que los docentes de todos los niveles $y$, en el caso que nos ocupa, el universitario, den el salto hacia prácticas innovadoras que pongan de manifiesto destrezas de pensamiento de nivel superior, conjuntamente, con actividades participativas que promuevan aprendizajes significativos. Es muy necesario que el profesor se desprenda del supuesto de que debe ser el epicentro de los contenidos. La idea es promover la eficacia hacia los niveles cognitivos y desarrollo de habilidades sociales en las que los estudiantes se empoderen de la construcción de conocimientos, en ambas vertientes: individual y grupal. En el caso de los docentes, han de moverse de un modelo de "transmisión de conocimiento" o "centrado en el profesor" a un modelo de "facilitación de los aprendizajes" o "centrado en el aprendizaje" (Gallargo, Sánchez, Ros \& Ferreras, 2010). Por lo tanto, se deben ir modificando los roles en el ambiente áulico.

En cuanto a la metodología innovadora en torno a la cual gira este trabajo, el método de Clase Invertida o Flipped Classroom (FC), forma parte del repertorio del diseño de estrategias de enseñanza novedosas que potencian el desarrollo de pensamiento crítico, resolución de problemas, la creatividad, entre otros. Es decir, al cambiar la dinámica de trabajo, los estudiantes pueden reflexionar, de forma individual, sobre los conceptos y, una vez en el aula, "debatir sobre esas reflexiones con sus compañeros, intercambiar impresiones, intentar llegar a una solución o interpretación conjunta, formar nuevas perspectivas, etc" (García-Barrero, 2013, p. 5).

El aula inversa también puede ayudarnos en una labor que potencie la participación activa de los estudiantes, y alude a un cambio de rol del profesor, quien se convierte en co-aprendiz y co-investigador, a través de 
invertir el proceso tradicional de la clase. Sánchez, Ruiz y Sánchez (2014) afirman que:

La clase invertida básicamente consiste en emplear el tiempo fuera del aula en realizar determinados procesos de aprendizaje que tradicionalmente se hacen dentro de la misma $y$, por su parte, dentro del aula, con la presencia, guía y experiencia del docente, el tiempo se emplea en potenciar y facilitar otros procesos de adquisición y práctica de conocimientos ( $p$. $3)$.

A manera de ejemplo, en la clase tradicional el profesor imparte charlas magistrales y asigna, para la casa o de tarea, actividades con el fin de que los estudiantes "refuercen" lo aprendido en clase. En contraposición, en el FC, las charlas y conferencias del profesor son sustituidas por videos, documentos, infografías, que el aprendiente va analizando y verificando sus contenidos para dedicar tiempo suficiente en la clase a actividades productivas que generen conocimiento de altos niveles de pensamiento.

Otro elemento característico de este método es que, con una consigna de trabajo bien diseñada, desde el punto de vista didáctico, provoca que los estudiantes vayan adquiriendo cada vez más responsabilidad acerca de sus propios aprendizajes. Pero para lograr que esto se de manera efectiva, Fernández (2016) enfatiza en la "obligada conveniencia de explicar muy bien a los alumnos en qué consiste invertir la clase y en lo importante que es que sigan el guión a rajatabla" (pág. 10).

Cuando los estudiantes conocen de qué trata la consigna, qué se espera de ellos y cómo serán evaluados, procuran asumir su rol protagónico para alcanzar el éxito en el proceso.
Esta metodología permite, además, mayor interacción entre los estudiantes y hace más potable el seguimiento personalizado por parte del profesor, quien se convierte en guía y facilitador del proceso. De acuerdo con Serrano y Pons (2011), el profesor funge como "mediador entre la actividad constructiva del alumno y los contenidos, posibilita la construcción de representaciones cognitivas de estos últimos adaptadas a las metas instruccionales" ( $p$. 23). Con este método se busca que ni uno solo de los estudiantes se quede sin conocer sobre el tema y poder interactuar con los demás. Una de las técnicas, entre el amplio abanico de posibilidades que se puede usar es la de peer instruction. Esta estrategia consiste en la reunión de dos o tres estudiantes, donde se socializa o discute sobre lo que entienden y qué conocen acerca de un tema en particular (Santiago, 2014).

Como una vía por excelencia, para lograr el FC, se hace uso del blended learning (B-learning o aprendizaje combinado). Garrison y Vaughan (2008) lo definen como una fusión entre las clases presenciales, con la comunicación cara a cara y la información escrita o visual que se presenta en línea. Lo anterior sugiere que se combine una gama de recursos y estrategias que pueda tener mucho mayor impacto en los aprendizajes que cuando se utiliza uno solo de los métodos.

Dado que, como docentes, si bien es cierto que "no podemos 'diseñar' los aprendizajes" (Bartolomé, 2004, pág. 19), sí podemos delinear nuestras metodologías de enseñanza y la incorporación del B-learning puede aumentar la motivación de los estudiantes, así como la ampliación del espectro de tácticas didácticas (Vera, 2008). No obstante, hay ciertas condiciones que inciden al momento de seleccionar materiales y herramientas de estudio para que el abordaje de los contenidos sea lo más viable y adecuado. Por esto, la figura 1, a continuación, presenta 4 criterios que, según Brennan (2004) han de tenerse en cuenta.

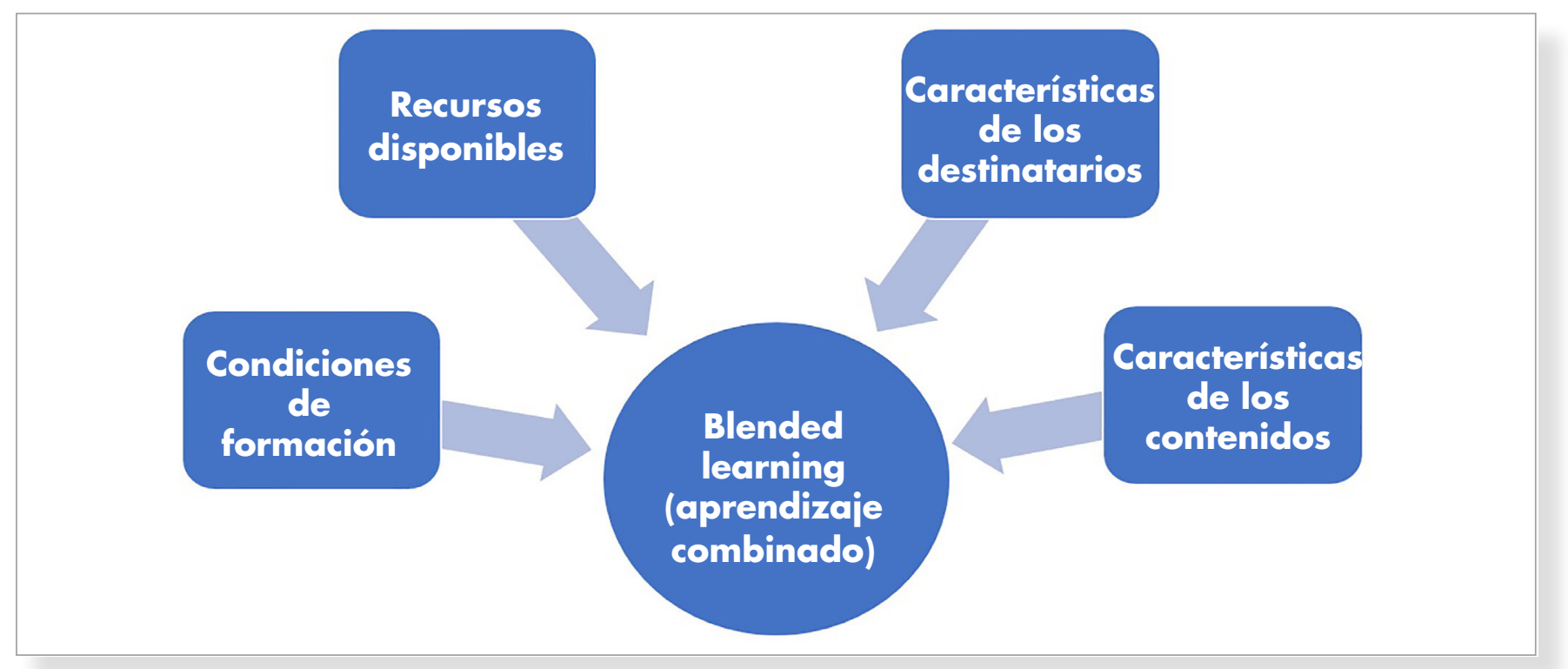

Figura 1 Criterios de selección de recursos para Blended Learning

«El b-learning y la clase invertida para el desarrollo del aprendizaje activo, la autogestión y el pensamiento crítico en el ámbito universitario», Jeanette Chaljub. Cuaderno de Pedagogía Universitaria Vol 13 / no. 26 / julio-diciembre 2016 / República Dominicana / PUCMM / ISSN 1814-4152 (en línea) / ISSN 1814-4144 (impresa) / p. 36-47 
Es decir, que para que se produzca una metodología combinada entre la clase presencial y los recursos y herramientas telemáticas, es necesario conocer qué hay disponible, cuál es el nivel de conocimiento de los estudiantes y sus estilos de aprendizaje. Además, se ha de verificar la disponibilidad y facilidad de uso de los dispositivos tecnológicos, así como el entramado de contenidos que se han de enseñar y aprender. De ahí, que la incorporación del aprendizaje combinado o blended learning (B-learning) es una "interesante estrategia que apunta a integrar las mejores prácticas pedagógicas con la última tecnología disponible para entornos virtuales de aprendizaje" (Vera, 2008, p. 13).

Es interesante destacar que el B-learning y el FC se sustentan, básicamente, en el aprendizaje ubicuo, un aprendizaje que se puede desarrollar 'en todo momento y en cualquier lugar'. Sobre esto, Burbules (2014) refiere a cómo los límites tradicionales del aula se extienden en dos vías: los estudiantes trabajan de forma independiente en sus casas con distintos tipos de tarea; pero, además, la actividad en clase se ve matizada con nuevas metodologías de enseñanza que promueven estrategias de aprendizaje con enfoque constructivas.

\section{Recursos utilizados en la modalidad de b-learning}

Dentro de las tecnologías emergentes aplicadas a la Educación, se encuentran los recursos educativos abiertos (REA) muy utilizados en las clases, especialmente, en la metodología combinada. Butcher (2015) los define como "cualquier recurso educativo que esté plenamente disponible para ser usado por educadores y estudiantes, sin que haya necesidad de pagar regalías o derechos de licencia" (p. 5). Existe un gran abanico de posibilidades, entre las que se pueden mencionar los materiales didácticos en línea, vídeo, programas de infografías, mapas mentales y mapas conceptuales, rúbricas, tablas de conversiones, programas de juego (gamificación), encuestas, libros electrónicos, etc.

Como se ha explicado en las líneas anteriores, el B-learning permite una combinación de actividades didácticas que se realizan, tanto de manera presencial como online, buscando alternativas para que los estudiantes se puedan motivar y comprometer con el proceso. Por eso, es importante evaluar siempre los aprendizajes de los estudiantes como una forma de ir adecuando la dinámica de la clase. Esta estrategia es muy valiosa en el modelo de FC, ya que persigue ajustar los métodos de acuerdo a los aprendizajes individuales y grupales hasta la construcción de nuevos conocimientos.
Como se expresó en las líneas anteriores, dentro de las distintas herramientas tecnológicas para la educación, se encuentra la gamificación. Este nombre tiene su origen en la palabra "juego", en inglés, que es "game". De acuerdo con Marín Díaz (2015), no se debe confundir con ludificación educativa. La gamificación aborda los contenidos del programa de forma integrada para incrementar la motivación y, sobre todo, el desarrollo de la creatividad en los estudiantes y el mismo profesor. Se trata, pues, de trasladar los entornos regulares de juegos electrónicos a otros de diversas áreas, como es el caso de estrategias de enseñanzas alternativas. Sin embargo, Carpena, Cataldi y Muñiz (2012) sostienen que uno de los desafíos es "llevar esta propuesta al salón de clases sin que parezca una clase para divertirse o pasar el rato ni caer en la banalidad o en la demagogia hacia los estudiantes" (p. 634). La intención pedagógica ha de estar alineada con las competencias esperadas y todo el quehacer áulico ha de estar encaminado hacia los aprendizajes, incluyendo metodologías participativas y entretenidas, con miras a lograr la construcción de nuevos saberes.

Siguiendo esta línea de ideas, una manera de contrarrestar la falta de interés que presentan muchos estudiantes, y bajo el principio de la gamificación, se han creado varios dispositivos que ayudan a aumentar la motivación y la participación activa. Entre las primeras herramientas creadas con este fin fueron los clickers, que usan una especie de control remoto para que los estudiantes respondieran a preguntas desde sus propios móviles, siguiendo el sistema de respuesta personal (personal response system). Los clickers son ampliamente utilizados en las universidades. No obstante, presenta algunos requerimientos como: un software específico, captador de infrarrojos, número limitado de mandos, son costosos y requieren mantenimiento (Pintor, Madera, Herreros, \& López, 2014).

Otro programa similar, que fue creado en el año 2011 por el profesor Alf Inge Wang, de la Norwegian University of Science And Technologyse, en Noruega, es el Kahoot. A diferencia de los clickers, destaca su simplificación, pues su uso es a través del internet. No se requieren dispositivos especiales y es un REA. Es decir, además de gratuito, se puede utilizar en cualquier momento. Es una herramienta sencilla. Su descarga es muy fácil y amigable, tanto para los docentes como para los estudiantes.

"La actividad fundamental con esta herramienta se basa en el empleo de cuestionarios, encuestas y discusiones (denominados comúnmente kahoots), propuestas generalmente por el docente y que buscan propiciar una participación consciente de sus estudiantes" (Jaber, 2016 , p. 226). Las acciones a realizar las pueden 
diseñar los docentes y los estudiantes. Como se explicó en el apartado anterior, no se trata solo del juego como un entretenimiento. Es, más bien, una herramienta interactiva que permite potenciar los aprendizajes y la participación activa de los estudiantes. Es una forma muy enriquecedora de propiciar la discusión y la conexión de nuevos saberes con los previos, en forma de juego, desde los mismos aparatos móviles que suelen utilizar los estudiantes. Corroborando lo expuesto por Jaber, Rodríguez (2017) alude a esta aplicación como un recurso que permite encuestas, cuestionarios y discusiones. Sobre todo, cabe destacar que facilita la retroalimentación en tiempo real, propiciando un ambiente de aprendizaje agradable y cómodo, dando información al docente de cómo van los aprendizajes de los estudiantes.

\section{Aspectos metodológicos}

La experiencia piloto que se llevó a cabo se trata de la clase invertida y b-learning dentro de las asignaturas de Neurociencias e Investigación Educativa de la Carrera de Educación de la Pontificia Universidad Católica Madre y Maestra. Cabe destacar que solo se trató un tema en particular para cada una de las asignaturas, de forma puntual, para, de una manera exploratoria, empezar a implantar esta metodología, de forma más sistemática. No se contempló como rutina de trabajo en las asignaturas.

Este estudio es de corte transversal, llevado a cabo durante el período académico 1-2016-2017, en los meses de octubre y noviembre. Su alcance es interpretativo, en el marco de la innovación pedagógica, destacándose principalmente dos competencias específicas dentro del Bloque de Formación Básica para el ejercicio de la docencia: Gestión de los aprendizajes e Investigación e Innovación Educativas.

La estrategia consistió en que los estudiantes realizaran lecturas individuales de los documentos asignados y observaran videos cuyos contenidos están relacionados con los contenidos de la asignatura, previamente a la clase. Para definir los conocimientos individuales y promover la gamificación, se utilizó el programa Kahoot.

Dentro de la cronología o secuenciación de la clase invertida, se dieron los siguientes pasos:

- Como asignación individual, se propuso una lectura previa de material didáctico para cada uno de los temas a tratar, los cuales se explican más adelante. Una vez realizada la lectura, cada estudiante elaboró un infograma y un breve ensayo argumentativo que subió al campus virtual, dentro de su espacio personal.
- Como asignación grupal, previo al encuentro, compartieron en el foro diseñado en el Campus Virtual, como debate sencillo, para exponer sus ideas y posturas acerca del tema y comentar sobre la de otro compañero de forma constructiva. La consigna de este foro, a grandes rasgos, se enfocó en que el estudiante pudiera exponer su idea sobre el tema central de la lectura y una opinión de la misma. Luego, debía escoger una entrada de dos de sus compañeros para corroborar o contradecir las exposiciones. Todo lo anterior acompañado de los argumentos expuestos en su ensayo y sustentados con una o dos de las fuentes que habían utilizado. Aquí se trabajan las habilidades superiores de pensamiento, pues están analizando distintas partes del artículo. Además, están construyendo, como grupo, sus propios conceptos de los temas que se van a desarrollar en el aula.

- Si la docente, como dinamizadora del foro, notaba que los estudiantes se salían del hilo conductor, realizaba preguntas disparadoras para reorientar la discusión.

- El foro, además, contenía un vídeo relacionado a la lectura previa que fue analizado por los estudiantes. Esto le permitió conectar la reflexión individual y los comentarios en el foro.

- Una vez en clase, se estableció un tiempo con el fin de aclarar dudas e inquietudes y verificar los aprendizajes individuales y adecuar los andamiajes para, luego, proceder a la realización de peer instruction o aprendizaje entre iguales con el fin de trabajar en una serie de interrogantes para la resolución de la tarea. Esta última consistió en hacer un mapa conceptual (síntesis), en una asignatura, y un muñeco tridimensional en otra, en los que se describían las partes esenciales de los artículos y vídeos estudiados (síntesis y creación de nuevos saberes). Una parte importante es que ellos mismos, dentro de los grupos que les tocó trabajar, pudieron crear una estrategia nueva para los temas tratados.

- Además del contenido propio de la asignatura, los estudiantes mediante consenso y búsqueda de información, debatieron para tomar decisiones en cuanto al desarrollo de unidades de clase integrando la metodología de clase invertida y elaboraron diferentes propuestas de cómo pueden implementar este método en sus planificaciones como futuros docentes. Como se puede notar, también, en esta parte hay desarrollo de pensamiento crítico ya que se trata de, luego, de un análisis sobre el material de estudio, la puesta en común, las orientaciones del profesor, releerlo (si es necesario), buscar 
información en otras fuentes, para que luego se formulen nuevas ideas para estrategias de enseñanza en el marco del ámbito educativo para el cual se están desarrollando.

- Para esto, se conformaron grupos heterogéneos de cuatro miembros, donde cada uno aportó sus aprendizajes previos, lo que entendió y pudo extraer de la lectura y del vídeo, además de las participaciones en el foro.

- En plenaria, expusieron los resultados con dibujos, esquema, mapas conceptuales. (sistematización del conocimiento personal y social, Gairín, 2007).

- Como cierre de la clase, se reforzaron los conceptos, tanto de las exposiciones de los grupos como un resumen por parte de la docente de las ideas que se iban presentando en plenaria y aquellas que, de alguna forma, no lograron desarrollarse en su totalidad. Un aspecto relevante en esta fase de cierre es que la docente presentó un resumen de las participaciones en el foro como forma de recapitular los conceptos.

- Ya que, en la clase invertida, como toda metodología activa, es importante tanto el proceso como el producto, para los aprendizajes, se procedió a realizar el Kahoot que contiene preguntas de selección múltiple relacionados a los temas desarrollados en clase para que los estudiantes vayan respondiendo y se puedan ir retroalimentando los resultados. La figura 2 que sigue muestra una parte de las preguntas realizadas en el Kahoot para la evaluación en el cierre de la clase de Neurociencias aplicadas a la Educación.

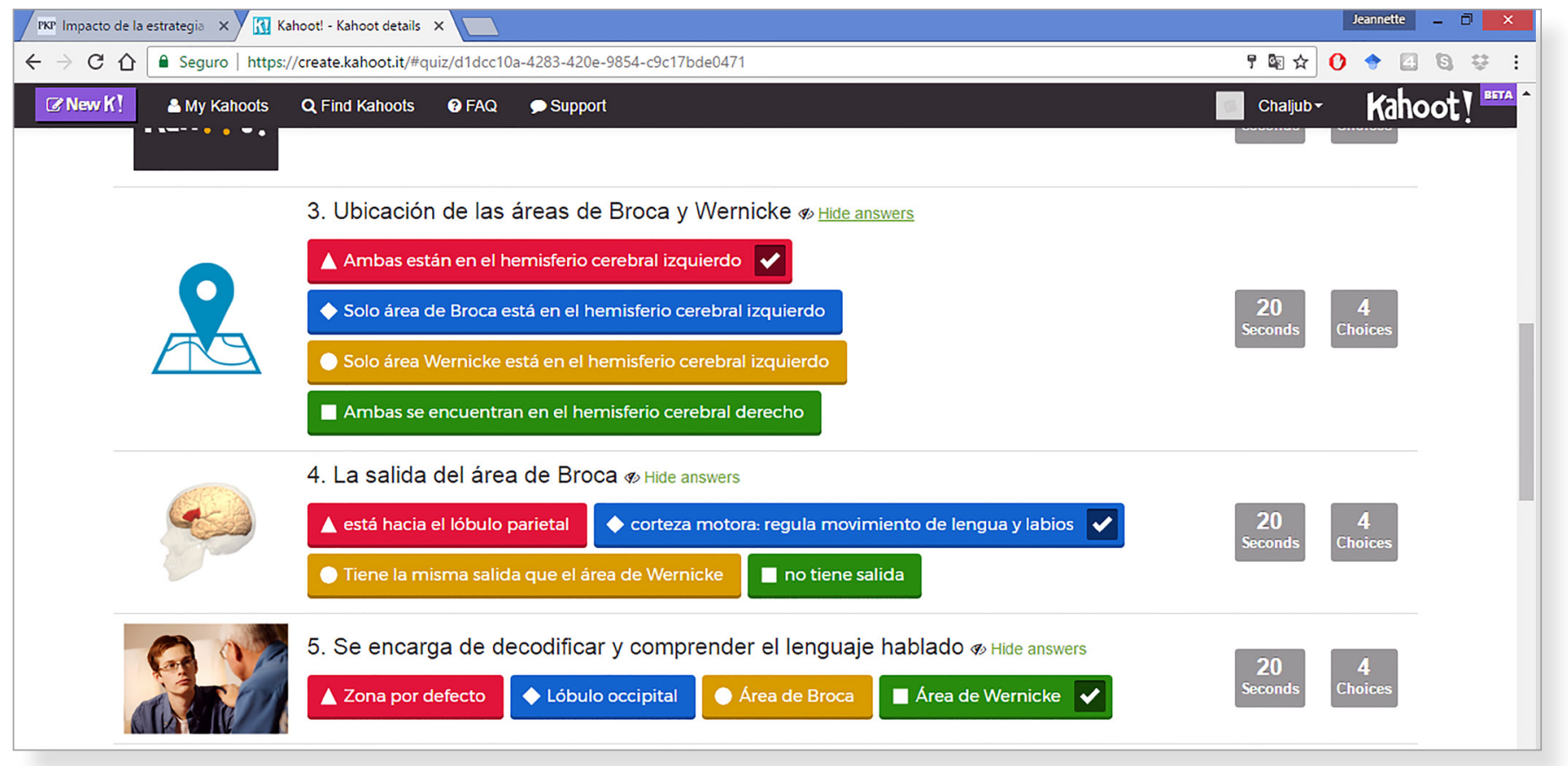

Figura 2 Modelo de preguntas para Kahoot

Como puede observarse de la figura anterior, este programa es un sistema que promueve que los estudiantes respondan por control remoto. En la pantalla, se muestra la pregunta, por ejemplo: Ubicación de las áreas de Brocca y Werncike (solo sale esto en la pantalla proyectada. En los móviles, ipads y portátiles, a los estudiantes les salen las opciones de las preguntas (los cotejos en las respuestas correctas, no los pueden ver los estudiantes. Esto es desde el enlace del maestro).

En este caso, el tiempo máximo para responder fueron 20 segundos, como aparece en el lateral derecho. Los estudiantes marcan la respuesta que crean correcta, previamente establecidas por el profesor.
De las líneas anteriores, tanto la metodología invertida, con el uso del b-learning, como el trabajo colaborativo promueven el desarrollo de habilidades superiores y las competencias esperadas. A saber:

- En la asignatura de investigación Educativa, el tema fue "Métodos de investigación". Con la FC, los estudiantes, una vez dominados los conceptos y discutidos en la fase de inicio de la clase (fase de conocimiento), pudieron evaluar distintos tipos de planteamiento de problema presentados por sus pares (fase de evaluación), a través de la discusión en el foro, y luego elaboraron preguntas de investigación 
y tomaron decisiones sobre el tipo de metodología que se debía aplicar en cada caso (fase de creación).

- En Neurociencias aplicadas a la Educación, el tema desarrollado fue el de "Los procesos mentales". En este caso, ya los estudiantes habían estudiado en clase los fundamentos y realizado infogramas para la fase de comprensión, los que fueron compartidos y debatidos en el foro. Al momento de la FC, elaboraron una unidad de clase, de acuerdo a su concentración, conectándola con los procesos mentales y con las distintas partes del cerebro, enfatizando en el diseño de actividades didácticas significativas (construcción de nuevas ideas).

La población de este estudio la constituyen los estudiantes de la carrera de Educación de la PUCMM y la muestra está compuesta por 31 estudiantes de la asignatura de Neurociencias y 14 de la asignatura de Investigación Educativa, para un total de 44 estudiantes, de los que respondieron solo 40. Los demás estaban ausentes el día de la actividad.

Una vez concluido el proceso, se invitó, de manera voluntaria a los estudiantes, a completar un cuestionario sobre su satisfacción y nivel de percepción de la clase invertida en cuanto a los aprendizajes previos, el ambiente de trabajo de aula, intercambio de opiniones, etc.

El cuestionario sobre la apreciación de la metodología invertida y el uso del Kahoot, dentro del proceso de gamificación de los estudiantes consta de 8 ítemes, divididos en cuatro categorías, como sigue:

- Material didáctico

- Metodología activa: su relación con el desarrollo de pensamiento crítico

- Construcción de nuevos saberes

- Valoración de la clase invertida y del Kahoot

Se le dio una valoración de 1 al 5 en la escala Tipo Likert, con la siguiente leyenda:

- $\quad 1$ es TOTALMENTE EN DESACUERDO

- 2 es EN DESACUERDO

- 3 es MEDIANAMENTE DE ACUERDO

- 4 es DE ACUERDO

- 5 es TOTALMENTE DE ACUERDO

Además, al final, se colocó una pregunta abierta: ¿Qué cambiaría de este método?

\section{Resultados}

Para la valoración general de la experiencia de la clase invertida (flipped classroom), el total de los estudiantes participantes la encontró como una experiencia "positiva", estando el $90 \%$ de ellos totalmente de acuerdo, y solo el $10 \%$, afirmó estar de acuerdo, como se refleja en la siguiente figura:

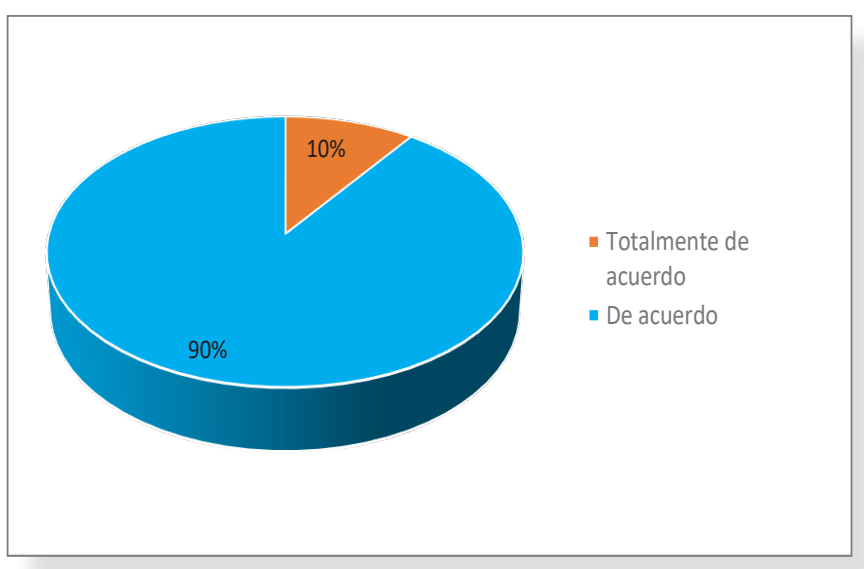

Figura 3 Valoración de la experiencia de Clase Invertida

Algunos de los estudiantes tuvieron inconvenientes con el móvil, pues el programa les dejó de funcionar en la mitad de la actividad, a pesar de haber hecho una prueba antes y otros no vieron el vídeo de estudio autónomo, por lo que no conocían algunas de las preguntas.

No obstante, se les permitió que respondieran en grupo, porque el programa así lo permite. Por consiguiente, de 40 estudiantes, 39 respondieron estar totalmente de acuerdo en que el Kahoot es herramienta de gamificación, fácil de descargar y de usar, y ayuda a que los aprendizajes se realicen de manera divertida e innovadora.

Otro de los hallazgos importantes es que un 93\% de los estudiantes está totalmente de acuerdo que prefiere las metodologías activas en vez de las charlas magistrales. Mientras que un $5 \%$ de ellos, está medianamente de acuerdo y tan solo el $2 \%$, contestó que está de acuerdo, como se puede observar en la figura 4.

Esto es un dato muy relevante, sobre todo, que los participantes en este ejercicio exploratorio están en su formación inicial para prepararse como futuros docentes. Por lo tanto, es de vital importancia que nosotros, como formadores de formadores, les enseñemos estrategias interesantes e innovadoras que potencien los aprendizajes. 


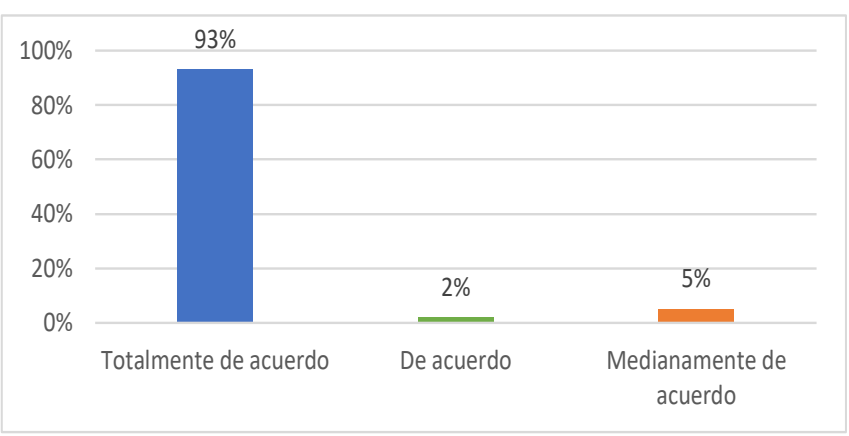

Figura 4 Preferencia de metodologías activas Vs. Charlas magistrales

Uno de los componentes que destaca la clase invertida es que los estudiantes, de manera individual y previo a la clase, pueden desarrollar las habilidades inferiores de pensamiento (conocimiento, comprensión y aplicación del conocimiento obtenido para llevar a cabo procesos algorítmicos) a través de la consigna de trabajo, guías de lectura y análisis de vídeos para luego compartir esos conocimientos con sus compañeros y, así, poder crear nuevos saberes. Por lo tanto, se aprovecha la clase presencial para potenciar, de forma colaborativa, las habilidades superiores de pensamiento o pensamiento crítico (análisis, síntesis, evaluación, creación).

Con relación a este aspecto y la percepción de los estudiantes, 35 de ellos (88\%) estuvieron totalmente de acuerdo en que, al realizar la metodología activa en clase, después del aprendizaje autónomo, pudo desarrollar sus propios aprendizajes, construir nuevas ideas y compartir con sus compañeros, cada uno aportando lo que, de manera individual, aprendió.

El resto, 12\%, también manifestó estar de acuerdo. Esto, además, incluye, el desarrollo de competencias como pensamiento crítico, negociación, argumentación y creatividad, como lo muestra la Tabla 1 a continuación.

\section{Tabla 1}

Percepción de los estudiantes respecto a sus aprendizajes a través de la Clase Invertida

\begin{tabular}{|l|c|c|}
\hline \multicolumn{1}{|c|}{ Escala de valoración } & No. de Estudiantes & Porcentaje \\
\hline Totalmente de acuerdo & 35 & 88 \\
\hline De acuerdo & 5 & 12 \\
\hline Total de estudiantes & 40 & $100 \%$ \\
\hline
\end{tabular}

Como se puede observar en los datos obtenidos, los tres otros ítemes de valoración: medianamente de acuerdo, en desacuerdo y totalmente en desacuerdo, no fueron contemplados en las respuestas, lo que evidencia que este método es muy eficaz en los aprendizajes. Para eso, es necesario que el docente planifique de manera productiva la parte presencial de la clase. El espacio de trabajo en el aula debe contemplar los momentos esenciales para la producción de conocimientos y relevancia de los aprendizajes. Comenzar con la recapitulación de los objetivos de aprendizaje, dar seguimiento a lo aprendido y aclarar dudas e inquietudes sobre la asignación previa. Es decir, repasar y esclarecer aquello que no quedó tan claro, asegurarse de que si alguien no ha visto el vídeo o leído el material, propiciar un breve conversatorio. En fin, preparar la plataforma cognitiva para que, una vez en los grupos, puedan hacer sus aportes. En este sentido, la tabla 2 muestra que 32 de los 40 estudiantes participantes afirmaron estar totalmente de acuerdo en que recibieron el apoyo constante de su profesora, atendiendo a sus dudas e inquietudes, para la realización de la tarea autónoma y activación de conocimientos previos. De igual forma, 7 de ellos sostuvo que está de acuerdo y solo 1 mostró estar medianamente de acuerdo.

\section{Tabla 2}

Asistencia y orientación de la maestra en la preparación previa

\begin{tabular}{|l|c|c|}
\hline \multicolumn{1}{|c|}{ Escala de valoración } & No. de Estudiantes & Porcentaje \\
\hline Totalmente de acuerdo & 32 & 80 \\
\hline De acuerdo & 7 & 18 \\
\hline Medianamente de acuerdo & 1 & 2 \\
\hline Total de estudiantes & 40 & $100 \%$ \\
\hline
\end{tabular}


En cuanto a la pregunta abierta ¿qué cambiaría de este método?, refiriéndose a la Clase Invertida y al Kahoot, de una forma casi universal, los estudiantes respondieron que "nada". Encontraron que la estrategia, les ayudó a concentrarse en el tema, que sus ideas eran tomadas en cuenta y que pudieron entender y conectar los conceptos a las propuestas y consignas de trabajo para los productos esperados. Sobre el Kahoot, la mayoría de las respuestas fue... iqué se use siempre!

Sin embargo, entre las limitaciones de este método se encuentran la resistencia que algunos estudiantes presentan en la participación en el foro. Les cuesta seguir las instrucciones de las consignas. Otro de los obstáculos que se presentaron en ambas experiencias, fue la lentitud del internet. Esto dificultaba el acceso de los estudiantes a la app que, en esos momentos, no respondía, por lo que no podían seguir con el juego y responder a las preguntas. Para que no se desmotivaran, la forma de compensar esta parte fue, poner la opción del Kahoot en grupos que permiten que más de uno pueda dar la respuesta desde un solo móvil.

Las diferencias en las respuestas al inicio en la recogida de saberes previos sobre los materiales de lectura, el análisis del vídeo y participación en el foro y aquellas con relación al proceso completo de la clase invertida y el uso del Kahoot, se pueden considerar que son mínimas en cuanto a la fundamentación base de conocimiento y comprensión del contenido. Lo que sí se puede evidenciar es la calidad de las respuestas. Es decir, los argumentos fueron más amplios, conectados con los conocimientos que ya poseían. El Kahoot, de forma particular, evidenció la asertividad en las respuestas de los estudiantes ya que este mide, tanto la calidad de los aprendizajes, como el tiempo en que se responde.

Con relación a cómo contestaron los estudiantes a las preguntas del Kahoot, en los gráficos que siguen, se mostrará, a modo de ejemplo, las respuestas de los estudiantes a las 3 preguntas que se expusieron en la figura 2 .

Para el caso de la pregunta "Ubicación de las áreas de Brocca y de Wernicke", los 31 estudiantes de la asignatura Neurociencias aplicada a la Educación respondieron correctamente: ambas se encuentran en el hemisferio izquierdo. Lo mismo sucedió con el caso de la pregunta "Se encarga de decodificar y comprender el lenguaje hablado", todos los estudiantes respondieron correctamente y afirmaron que es el área de Wernicke.

Para el caso de la "salida del área de Brocca", la figura 5 muestra los siguientes resultados.

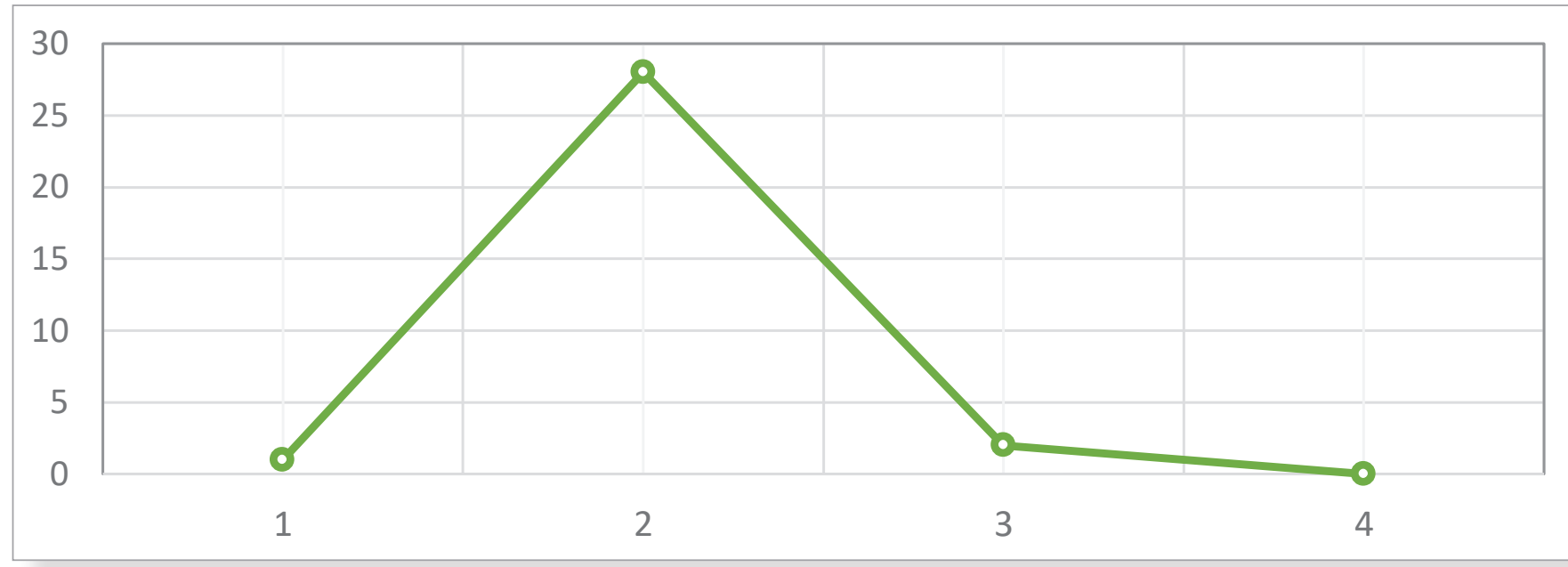

Figura 5 Asistencia y orientación de la maestra en la preparación previa

\begin{tabular}{|l|l|}
\hline \multicolumn{2}{|c|}{ Leyenda numeración respuestas sobre salida del área de Brocca } \\
\hline 2 & Está hacia el lóbulo parietal \\
\hline 3 & Corteza motora: regular movimientos de labios y lengua \\
\hline 4 & Tiene la misma salida que el área de Wernicke \\
\hline
\end{tabular}

«El b-learning y la clase invertida para el desarrollo del aprendizaje activo, la autogestión y el pensamiento crítico en el ámbito universitario», Jeanette Chaljub. Cuaderno de Pedagogía Universitaria Vol 13 / no. 26 / julio-diciembre 2016 / República Dominicana / PUCMM / ISSN 1814-4152 (en línea) / ISSN 1814-4144 (impresa) / p. 36-47 
La figura 5 muestra claramente que 28 de los 31 estudiantes que participaron para la asignatura de Neurociencias aplicadas a la Educación respondieron correctamente, con ligeras diferencias en el tiempo que duraron para responderlas. Uno solo respondió que está en el lóbulo parietal y otro de ellos, respondió que tiene la misma salida que el área de Wernicke.

Ninguno de los estudiantes respondió que el área de Brocca "no tiene salida", lo que conduce a pensar que están bastante claros de la función de esta parte de nuestro cerebro cuya función es la producción adecuada del acto del habla.

\section{Conclusión}

En síntesis, debemos resaltar que el método de Clase Invertida o Flipped Classroom requiere etapas muy bien diseñadas para la construcción de conocimientos y consecución de aprendizajes de alto nivel, pero el resultado es eficaz. Los estudiantes se sienten motivados cuando ellos mismos pueden compartir conocimientos, crear ideas nuevas, colaborar con sus pares. Esto porque se invierte lo que tradicionalmente era usado para charlas y clases de exposición por parte del profesor a actividades que realmente se relacionan con los aprendizajes como son: análisis, aprendizaje por descubrimiento, decisiones grupales, etc. (García-Barrero, 2013).

En cada una de las actividades de Clase Invertida se evaluaron los aprendizajes a través de la gamificación, con el Kahoot, quedando evidenciado cómo se elevan los niveles de protagonismo por parte de los estudiantes y deseos de aprender. Es importante puntualizar que las actividades didácticas que requirieron de un nivel más profundo de pensamiento, se realizaron en clase, con la guía del docente, bajo estructuras generadoras de desarrollo del pensamiento crítico a través de un aprendizaje participativo.

Para corroborar lo anterior, se ha de pensar en una redefinición de los aprendizajes ya que no se trata solo de acumular saberes, sino más bien de gestionar conocimientos, plantearse nuevos problemas, proponer soluciones alternativas, lo que lleva a la toma de decisiones sobre el propio conocimiento y el de los demás (Tourón, Santiago \& Díez, 2014).

Consideramos que lo más importante de incluir metodologías innovadoras como el $\mathrm{FC}$ es que el docente realice una planificación pensada en las fases de las habilidades cognitivas. Su objetivo será asegurar que aquellas que requieren un nivel inferior de pensamiento, que puedan ser ejecutadas sin la ayuda del maestro, el estudiante las realice de forma independiente. Con esto coinciden, Soler, Solanas, Aymerich y Brugada (2011, s/p) cuando afirman que la autogestión del conocimiento se concibe como "el marco en el cual el estudiante es el principal responsable y administrador autónomo de su proceso de aprendizaje".

Por lo tanto, requiere de un diseño pormenorizado de las actividades a implementar por los estudiantes, promoviéndose un cambio en el rol de ellos y en el del profesor, el cual girará en torno a las necesidades individuales de los estudiantes desde la fase del estudio independiente hasta la conclusión de los productos esperados, con el fin de optimizar sus aprendizajes.

En otro orden, los resultados arrojados en este estudio evidencian la necesidad de la formación docente en temas como la innovación pedagógica. Repensar en metodologías alternativas para los aprendizajes activos, con la combinación de la tecnología para potenciar la construcción de conocimientos.

Una idea que podría apuntar hacia la mejora de las clases, sería continuar este estudio con una serie de propuestas sistematizadas con el fin de realizar una investigación acción y llevarla a otros escenarios y disciplinas dentro de la universidad. Es más, se recomienda, crear comunidades profesionales de aprendizaje para el análisis y discusión de los resultados que se vayan generando, así como el intercambio de experiencias innovadoras en la clase invertida y la profundización en la gamificación.

\section{Referencias}

Bartolomé, A. (2004). Blended learning. Conceptos Básicos. Píxel-Bit. Revista de Medios y Educación. 23, 7-20.

Brennan, M. (2004). Blended learning and business change. Chief of Learning office Magazine.

Burbules, N. C. (2014). Los significados del 'aprendizaje ubicuo'. Archivos analíticos de Políticas Educativas, 22 (104).

Butcher, N. (2015). Guía Básica de Recursos Educativos Abiertos (REA). (2da. ed.). Francia: UNESCO.

Carpena, N., Cataldi, M. \& Muñíz, G. (2012). En busca de nuevas metodologías y herramientas aplicables a la Educación. Repensando nuestro rol docente en las aulas. 
Confederación de Empresarios de Navarra (2012). Competencias profesionales del Siglo XXI. Recuperado de: http://ifuturo.org/informes/ Competencias $\% 20$ Profesionales $\% 20$ para $\% 20$ el\%20Siglo\%20XXI.pdf

Domínguez, L. (2015). Impacto de la estrategia de aula invertida en el ambiente de aprendizaje en cirugía: una comparación con la clase magistral. Biomédica. 35, 513-521 doi: http://dx.doi.org/10.7705/ biomedica.v35i4.2640

Fernández, I. (coord.). (2016). Todo comienza lejos del aula. The flipped classroom Newsletter. 3-16.

Gallargo, B., Sánchez, F., Ros, C. \& Ferreras, A. (2010). Estilos docentes de los profesores universitarios. La percepción de los alumnos de los buenos profesores. Revista Iberoamericana de Educación. 51(4).

Gairín, J. (2007). Competencias para la gestión del conocimiento y aprendizaje. Cuadernos de Pedagogía. 370, 24-27

García-Barrero, A. (2013). El aula inversa: cambiando las repuestas a las necesidades de los estudiantes. Revista de la Asociación de Inspectores de Educación de España. 19, 1-8.

Garrison, D. \& Vaughan, N. (2008). Blended learning in Higher Education. Framework, principles, and guidelines. San Francisco, United States: JosseyBass.

Jaber, J. (coord.). (2016). Empleo de Kahoot como herramienta de gamificación en la docencia universitaria. III Jornadas Iberoamericanas de Innovación Educativa en el ámbito de las TIC Las Palmas de Gran Canaria.Recuperadode:http://acceda.ulpgc.es/ bitstream/10553/20472/1/0730076_00000_0032.pdf

Maldonado, M. (2007). El trabajo colaborativo en el aula universitaria. Laurus. 13(23), 263-278.

Maquilón Sánchez, J. J.; Mirete Ruiz, A. B.; García Sánchez, F.A.\&HernándezPina, F. (2013). Valoración de las TIC por los estudiantes universitarios y su relación con los enfoques de aprendizaje. Revista de Investigación Educativa, 31 (2), 537-554. http:// dx.doi.org/10.6018/rie.31.2.151891

Marín Díaz, V. (2015). La gamificación educativa. Una alternativa para la enseñanza creativa. Recuperado de:C:/Users/Jeanette/Downloads/13433-24540-1-PB.pdf
McAlpine, L. (2004). Designing learning as well as teaching. A research-based model for instruction that emphasizes learner practice. Active Learning in Higher Education, 5(2), 119-134.

Pérez de M.I., Bustamante, S. \& Maldonado, M. (2007). Aprendizaje en Equipo y Coaching en Educación. Una experiencia Innovadora. Publicación en extenso en Memoria de VII Reunión Nacional de Currículo y I Congreso Internacional de Calidad e Innovación en Educación Superior. Universidad Simón Bolívar.

Prieto, L. (2006). Aprendizaje activo en el aula universitaria: el caso del aprendizaje basado en problemas. Miscelanea Comillas. 64 (124), 173196.

Rodríguez-Fernández, L. (2017). Smartphones y aprendizaje: el uso del Kahoot en el aula universitaria. Revista Mediterránea de Comunicación.

Sánchez, J., Ruiz, J. \& Sánchez, E. (2014). Las clases invertidas: beneficios y estrategias para su puesta en práctica en la educación superior. Repositorio Institucional de la Universidad de Málaga. Recuperado de: http://riuma.uma.es/xmlui/handle/10630/7821? show=full

Santiago, R. (2014). Peer instruction y concept test, una Buena combinación. Recuperado de http://www. theflippedclassroom.es/peer-instruction-y-concepttest-una-buena-combinacion/

Serrano, J. \& Pons, Ma. (2011). El Constructivismo hoy: enfoques constructivistas en educación. Revista Electrónica de Investigación Educativa. 13 (1), 1-27.

Tourón J., Santiago, R. \& Díez, A. (2014). The flipped classroom. Cómo convertir la escuela en un espacio de aprendizaje. Digital-text. Recuperado de https://books.google.com.do/ books? $\mathrm{hl}=\mathrm{es} \& \mathrm{r}=\& \mathrm{id}=$ YWPPBAAAQBAJ\&oi= fnd \&pg $=$ PT $2 \& d q=$ clase + invertida $+0+$ flipped + classroom \&ots $=k V 63 \mathrm{MgriNU} \& \operatorname{sig}=A 40 R 3$ IkYj2ofN9gnOQEThBYnE74\#v=onepage \& $q=$ clase $\% 20$ invertida $\% 200 \% 20$ flipped $\% 20$ classroom\&f=false

Vera, F. (2008). La modalidad Blended Learning en Educación Superior. Recuperado de: http://sistemas2.dti.uaem.mx/evadocente/ programa2/Farm007_14/documentos/b-learning_ en_educacion_superior2008.pdf 